Discussion Paper No. 609

\title{
PORTFOLIO INERTIA UNDER AMBIGUITY
}

\author{
Takao Asano
}

June 2004

The Institute of Social and Economic Research Osaka University

6-1 Mihogaoka, Ibaraki, Osaka 567-0047, Japan 


\title{
Portfolio Inertia under Ambiguity*
}

\author{
Takao Asano $^{\dagger}$ \\ Institute of Social and Economic Research \\ Osaka University \\ Mihogaoka 6-1, Ibaraki, Osaka 567-0047 \\ JAPAN
}

First Version: August 1, 2001

This version: December 1, 2003

\begin{abstract}
We consider individual's portfolio selection problems. Introducing the concept of ambiguity, we show the existence of portfolio inertia under the assumptions that decision maker's beliefs are captured by an inner measure, and that her preferences are represented by the Choquet integral with respect to the inner measure. Under the concept of ambiguity, it is considered that a $\sigma$-algebra is not necessarily an appropriate collection of events to which a decision maker assigns probabilities. Furthermore, we study the difference between ambiguity and uncertainty by considering investors' behavior.
\end{abstract}

Journal of Economic Literature Classification Numbers: D81, G11

Key Words: Ambiguity, Knightian Uncertainty, Portfolio Inertia

${ }^{*}$ I would like to thank Hiroyuki Ozaki for his discussions and comments on this work. I am grateful to seminar participants at Keio University, Tokyo Institute of Technology, University of Tokyo, and Yokohama National University as well as participants at the conference on "Knightian Uncertainty" held at University of Tokyo. I am also grateful to Hiroyuki Kojima, Motonari Kurasawa, Daisuke Oyama, and Satoru Takahashi as well as participants at the 2002 Annual Meeting of the Japanese Economic Association in Otaru. Finally, I am greatly indebted to Akihiko Matsui, who patiently read through earlier versions of the present paper, encouraged me through uncountable many meetings, and gave me invaluable comments and suggestions. Needless to say, I am responsible for any remaining errors.

${ }^{\dagger}$ e-mail: tasano@iser.osaka-u.ac.jp 


\section{Introduction}

In stock markets, we often observe portfolio inertia, i.e., a situation in which some stocks are not traded or not priced for a few minutes or longer. This is neither an exceptional situation in which some stock price soars too high to be priced, nor the one where some stock price plummets too much to be traded. Now, a question is worth raising; what leads to portfolio inertia?

Under the standard expected utility theory, Arrow (1965) shows that an investor has the unique striking price, i.e., the price above which she is willing to sell, and below which she is willing to buy. Portfolio inertia generically exists only if the striking price for selling and that for buying have a spread. The standard expected utility theory does not account for the existence of the spread, except for a transaction cost, which is too small for institutional investors to justify the existence of portfolio inertia.

Dow and Werlang (1992) account for the existence of portfolio inertia under the Choquet Expected Utility (henceforth CEU) with convex non-additive measures. In the literature on CEU, decision makers' (henceforth DM) attitude toward uncertainty is captured by the convexity of non-additive measures. ${ }^{1}$ However, the assumption of the convexity of non-additive measures is so strong that there exist possibilities that important economic problems cannot be fully accommodated as an example in this paper explains. In this paper, we weaken the convexity, and adopt a new concept, i.e., the concept of ambiguity. In this alternative setting, DM's attitude toward ambiguity, which is differentiated from risk and uncertainty, is captured by the super-additivity of non-additive measures. The super-additivity is intuitively appealing compared with the convexity of non-additive measures, it is weaker than the convexity, and it can accommodate the situations in which the convexity cannot. The purpose of this paper is to account for the existence of portfolio inertia by adopting the concept of ambiguity.

In order to illustrate the point, suppose that an investor considers trading the stock of a multinational corporation that does business in two countries, Japan and the United

\footnotetext{
${ }^{1}$ For the definitions of non-additive measures and convexity, see Section 2 in details.
} 
States. We suppose that the company's profit is affected by the economic conditions of both countries. We also assume that the state of the world is described by one of the four elements in $S=\{g g, b g, g b, b b\}$, where the first and second letters stand for the economic conditions of Japan and the US, respectively. We assume that she assigns probabilities to the events that contain the state where the economic conditions of the two countries are good. However, we assume that she does not assign probabilities to the events where the conditions of the two countries are both good, and so forth. We make these assumptions in order to describe the situation in which investors do not have enough information to pin down whether the economic conditions of the two countries are in good, or in bad, or in opposite condition. Then the collection $\mathcal{A}$ of the events to which probabilities are assigned is $\mathcal{A}=\{S, \emptyset,\{g g, g b\},\{g b, b b\},\{b g, b b\},\{g g, b g\}\} .^{2}$ In the standard model, probabilities are assumed to be defined on a family of subsets of a state space, called a $\sigma$-algebra, while $\mathcal{A}$ is not. ${ }^{3}$ We call all subsets of $S$ outside $\mathcal{A}$ ambiguous events. As we explain in Subsection 2.2, there exists a situation in which the existence of portfolio inertia cannot be accounted for by the convexity of non-additive measures that is considered as capturing uncertainty aversion in the literature on non-expected utility theories. Before we provide the definition of ambiguity, let us review some of the existing concepts.

It is Ellsberg (1961) who first casts doubt on the validity of the Subjective Expected Utility (henceforth SEU) theory axiomatized by Savage (1954), which is widely applied to economic problems. ${ }^{4}$ Since then, a number of generalizations have been developed to overcome its shortcomings. ${ }^{5}$

In the course of development of non-expected utility theories, Knight's approach (1921) is reconsidered as one way to account for Ellsberg-type situations. According to Knight (1921), the risk situation is a situation where a probability measure can be

\footnotetext{
${ }^{2}$ See Subsection 2.2 in details.

${ }^{3}$ Note that $\mathcal{A}$ is not closed with respect to intersections.

${ }^{4} \mathrm{SEU}$ states that if a certain set of axioms is satisfied, then DM's beliefs are captured by a unique probability measure and her preferences are represented by the expected utility.

${ }^{5}$ Using an easy-to-understand example, Ellsberg shows that people often violate Savage's crucial axiom, the Sure Thing Principle.
} 
assigned, and the uncertainty situation is "all other situations." In the literature on nonexpected utility theories, Knight's risk situation is interpreted as a situation where DM's beliefs are captured by a unique probability measure, while the uncertainty situation is interpreted as a situation where DM's beliefs are not captured by a unique probability. We call an approach based on the latter interpretation the uncertainty approach. In the late 1990s, another approach based on a different interpretation of "all other situations" is proposed. This approach focuses on domains of probability measures, in other words, this considers that a $\sigma$-algebra is not necessarily an appropriate collection of events to which a DM assigns probabilities, while the other two focus on set functions by which DM's beliefs are captured. ${ }^{6}$ In the present paper, we call this approach the ambiguity approach.

The uncertainty approach is axiomatized and analyzed in the framework of the Maxmin Expected Utility (henceforth MMEU) theory and CEU. ${ }^{7}$ MMEU states that if a certain set of axioms is satisfied, then DM's beliefs are captured by a set of finitely additive measures and her preferences are represented by the minimum of expected utilities over the set of these measures. On the other hand, CEU states that if a certain set of axioms is satisfied, then DM's beliefs are captured by a non-additive measure and her preferences are represented by Choquet integrals. Unlike MMEU, CEU does not contain any axiom that captures DM's attitude toward uncertainty. ${ }^{8}$ Showing that CEU with a convex nonadditive measure is equivalent to a special case of MMEU, Schmeidler (1989) endows the meaning of uncertainty aversion with CEU. This enables us to analyze DM's behavior under uncertainty through the convexity of non-additive measures. ${ }^{9}$ Our understanding

\footnotetext{
${ }^{6}$ See Epstein (1999) and Zhang (1999)

${ }^{7} \mathrm{MMEU}$ is axiomatized by Gilboa and Schmeidler (1989) in the Anscombe and Aumunn (henceforth AA) framework and axiomatized by Casadesus-Masanell, Klibanoff and Ozdenoren (2000) in the Savage framework. CEU is axiomatized by Schmeidler (1989) in the AA framework and axiomatized by Gilboa (1987) in the Savage framework.

${ }^{8}$ See Axiom 5 (Uncertainty Aversion) in Gilboa and Schmeidler (1989).

${ }^{9}$ Let $\mu$ be a convex non-additive measure, $\mathscr{M}$ be the set of finitely additive measures on $\left(S, 2^{S}\right)$, and let $B(S, \mathbb{R})$ denote the space of bounded functions from $S$ into $\mathbb{R}$. Then

$$
\int X(s) \mu(d s)=\min \left\{\int X(s) P(d s) \mid P \in \operatorname{core}(\mu)\right\}
$$

where $X \in B(S, \mathbb{R})$ and the core of $\mu$ is the set of finitely additive measures on $\left(S, 2^{S}\right)$ that dominate
} 
of DM's behavior under uncertainty has been deepened by these new theories themselves and their applications to game theory (Dow and Werlang (1994), Lo (1996, 1998, 1999), and Marinacci (2000)), finance (Dow and Werlang (1992), and Epstein and Wang (1994, 1995)) or agency theory (Ghirardato (1994)).

Recently, Epstein (1999) casts doubt on the usage of the convexity of non-additive measures as uncertainty aversion, and introduces the concept of ambiguity. Epstein (1999) defines unambiguous events as those events to which a DM can assign probabilities, and ambiguous events as the ones to which she cannot. This corresponds to the second interpretation of Knight. Thus, we consider Epstein's approach as the ambiguity approach. One of the purposes of this paper is to seek the possibility of applications of the ambiguity approach to economic problems.

Zhang (2002) proposes a new axiomatization of CEU. This states that if a certain set of axioms is satisfied, then DM's beliefs are captured by an inner measure and her preferences are represented by the Choquet integral with respect to the inner measure. ${ }^{10}$ Zhang's axiomatization is more restrictive than Schmeidler's one. However, adopting Zhang's approach, we can incorporate the concept of ambiguity into CEU, and shed light on the possibility of its applications to other economic problems.

Based on Zhang (2002), we show that there exists portfolio inertia under CEU with an inner measure, which is neither additive nor convex. This approach is one of the starkest contrasts to the one that depends on the convexity of non-additive measures.

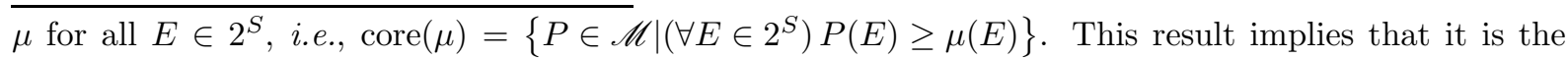
convexity of non-additive measures that serves the bridge between MMEU and CEU, and enables us to analyze DM's behavior under uncertainty through the Choquet integral. Mathematically, analyses of the Choquet integral are easier than those of the minimization of expected utilities over a set of measures in the framework of MMEU. For example, see Ghirardato (1997), and Ghirardato, Klibanoff and Marinacci (1998). The reason that the convexity of non-additive measures has been used in the literature on CEU and MMEU is this mathematical tractability of the Choquet integral and the meaning of uncertainty aversion through MMEU.

${ }^{10}$ If a certain set of axioms is satisfied, her preferences are represented as follows: there exists a nonconstant function $u: \mathcal{X} \rightarrow \mathbb{R}$ and a premeasure $p$ on $(S, \mathcal{A})$ such that

$$
(\forall f, g \in \mathcal{F}) \quad f \succeq g \Leftrightarrow \int u \circ f d p_{\mathcal{A}} \geq \int u \circ g d p_{\mathcal{A}},
$$

where $\mathcal{X}$ is some set of outcomes, $\mathcal{F}$ is some set of acts, and $p_{\mathcal{A}}$ is the inner measure corresponding to $(S, \mathcal{A}, p)$ and integrals are in the sense of Choquet integrals. For the definition of Choquet integrals, see Subsection 2.1. 
Furthermore, we study the reason that different investors have different degrees of portfolio inertia.

The organization of this paper is as follows. Section 2 provides an example, some mathematical tools including the concept of the Choquet integral, inner and outer measures, and $\lambda$-systems. Section 3 presents main results of the present paper. Section 4 concludes this paper. Proofs and mathematical results are relegated to Appendices.

\section{Preliminaries}

\subsection{Choquet Integrals}

Let $S$ be a set and let $2^{S}$ be the power set of $S$. A set function $\mu: 2^{S} \rightarrow[0,1]$ is a non-additive measure (or capacity) if $(a) \mu(\emptyset)=0$ and (b) $E, F \in 2^{S}$ and $E \subset F$ imply $\mu(E) \leq \mu(F)$, where $\emptyset$ denotes the empty set. A non-additive measure $\mu$ is convex if $\mu(E \cup F)+\mu(E \cap F) \geq \mu(E)+\mu(F)$ for all $E, F \in 2^{S}$.

Let $B(S, \mathbb{R})$ denote the space of bounded functions from $S$ into $\mathbb{R}$ and let $X \in$ $B(S, \mathbb{R})$. The integral of $X$ with respect to a non-additive measure $\mu$ is called the Choquet integral, and is defined by

$$
\begin{aligned}
& \int X(s) \mu(d s) \\
= & \int_{0}^{\infty} \mu(\{s \in S \mid X(s) \geq \alpha\}) d \alpha+\int_{-\infty}^{0}[\mu(\{s \in S \mid X(s) \geq \alpha\})-1] d \alpha,
\end{aligned}
$$

where integrals on the right hand side are in the sense of Riemann integrals. Throughout this paper, all the integrals are in the sense of Choquet integrals.

\subsection{An Example}

Suppose that an investor considers trading the stock of a multinational corporation that does business in two countries, Japan and the United States. We suppose that the company's profit is affected by the economic conditions of both countries. We also assume that the state of the world is described by one of the four elements in $S=\{g g, b g, g b, b b\}$, where the first and second letters stand for the economic conditions of Japan and the US, 
respectively. For example, $g b$ stands for the state where the economic condition of Japan is good and the economic condition of the US is bad. Let $X: S \rightarrow \mathbb{R}_{+}$denote the stock price of the company at $t=1$. Let $X(g g)=a_{1}, X(b g)=a_{2}, X(g b)=a_{3}$, and $X(b b)=a_{4}$. We assume that the company's profit is more likely to be affected by the economic condition of the US than that of Japan. So, we suppose that $a_{1}>a_{2}>a_{3}>a_{4}$. It is also assumed that she assigns probabilities to the events that contain the state in which the economic conditions of the two countries are good. However, we assume that she does not assign probabilities to the events in which the conditions of the two countries are both good, and so forth. We make these assumptions in order to describe the situation in which investors do not have enough information to pin down whether the economic conditions of the two countries are in good, or in bad, or in opposite condition. An interpretation is that she is unwilling to, even subjectively, judge the correlation between the two countries' economic conditions. We assume that she assigns $p_{1}$ and $p_{2}$ to $\{g g, g b\}$ and $\{g g, b g\}$, respectively. It is also assumed that $1-p_{1}$ and $1-p_{2}$ are assigned to $\{g g, g b\}^{c}=\{b g, b b\}$ and $\{g g, b g\}^{c}=\{g b, b b\}$, respectively. ${ }^{11}$ Thus, the collection $\mathcal{A}$ of the events to which probabilities are assigned is as follows:

$$
\mathcal{A}=\{S, \emptyset,\{g g, g b\},\{g b, b b\},\{b g, b b\},\{g g, b g\}\}
$$

The assigned values are given by

$$
\begin{gathered}
p(S)=1, p(\emptyset)=0, p(\{g g, g b\})=p_{1}, \\
p(\{g g, b g\})=p_{2}, p(\{b g, b b\})=1-p_{1}, p(\{g b, b b\})=1-p_{2} .
\end{gathered}
$$

We assume that she dislikes "ambiguity," in other words, she is very cautious, and that she uses a set function $p_{\mathcal{A}}: 2^{S} \rightarrow[0,1]$ given by

$$
p_{\mathcal{A}}(B)=\sup \{p(A) \mid A \in \mathcal{A}, A \subset B\}
$$

for every subset $B \in 2^{S}$, not necessarily in $\mathcal{A}$. ${ }^{12}$ This function assigns a number to every subset $B$, which is approximated from the inside by an "unambiguous" event $A \in \mathcal{A}$. Also

\footnotetext{
${ }^{11}$ We assume that $p_{1}$ and $p_{2}$ are in $(0,1)$.

${ }^{12} p_{\mathcal{A}}$ is a non-additive measure. See Lemma 1.
} 
assume that her preferences are represented by Choquet integrals with respect to $p_{\mathcal{A}}$, and that she is a CEU maximizer.

In the following situations, suppose that $p_{2}>p_{1} \cdot{ }^{13}$ First, consider a situation where she will buy the stock. Since the expected value of buying the stock is

$$
\int X(s) p_{\mathcal{A}}(d s)=\left(a_{2}-a_{4}\right) p_{2}+a_{4}
$$

the expected return from buying it is $\int X(s) p_{\mathcal{A}}(d s)-q$, where $q$ is the current price of the stock. Thus, if $q$ is less than $\int X(s) p_{\mathcal{A}}(d s)$, she will buy the stock. Next, consider a situation where she will sell the stock. Since the expected value of selling the stock is

$$
\int-X(s) p_{\mathcal{A}}(d s)=\left(a_{2}-a_{1}\right) p_{1}+\left(a_{3}-a_{2}\right) p_{2}-a_{3}
$$

the expected return from selling it is $q+\int-X(s) p_{\mathcal{A}}(d s)$. Thus, if $q$ is more than $-\int-X(s) p_{\mathcal{A}}(d s)$, she will sell the stock. Note that the next inequality holds:

$$
-\int-X(s) p_{\mathcal{A}}(d s)>\int X(s) p_{\mathcal{A}}(d s)
$$

Thus, if the current price $q$ is between these two numbers, she will not consider trading the stock. ${ }^{14}$ If this investor's beliefs are captured by a unique probability, in other words, if she does not care about ambiguity, then the above inequality is replaced by equality. Thus, under the standard model like Arrow (1965), the existence of portfolio inertia is not accounted for.

More observations are in order. A collection of the events to which probabilities are assigned is typically assumed to be a $\sigma$-algebra. In our example, however, since $\{g g, g b\} \cap\{g b, b b\}=\{g b\} \notin \mathcal{A}, \mathcal{A}$ is not a $\sigma$-algebra. Moreover, since

$$
p_{\mathcal{A}}(\{g g, g b\})+p_{\mathcal{A}}(\{g g, b g\})>p_{\mathcal{A}}(\{g g, g b, b g\})+p_{\mathcal{A}}(\{g g\})
$$

\footnotetext{
${ }^{13}$ For this inequality, we need assume that she believes that $\{g g, b g\}$ is more likely to happen than $\{g g, g b\}$. Even if the reverse inequality is assumed, portfolio inertia still exists.

${ }^{14}$ This phenomenon occurs when the expected values from buying and selling the stock are both negative, i.e.,

$$
\int X(s) p_{\mathcal{A}}(d s)-q<0 \text { and } q+\int-X(s) p_{\mathcal{A}}(d s)<0
$$
}


$p_{\mathcal{A}}$ is not convex.

In summary, we observe three points. First, a collection of the events to which this investor assigns probabilities is not a $\sigma$-algebra. Second, a set function that captures her attitude toward ambiguity is not a convex non-additive measure. Thus, such a situation as this example cannot be accounted for in the framework of Dow and Werlang (1992). Finally, even if the set function is not convex, portfolio inertia still exists.

\section{$2.3 \lambda$-systems, Inner Measures}

This subsection defines and discusses the concept of ambiguity, and provides mathematical results related to the concept. First of all, the definition of $\lambda$-systems is provided.

Definition 1. A non-empty class of subsets $\mathcal{A} \subset 2^{S}$ is a $\lambda$-system if

$(\lambda .1) S \in \mathcal{A}$,

( $\lambda .2) A \in \mathcal{A}$ implies $A^{c} \in \mathcal{A}$, and

$(\lambda .3)\left\langle A_{i}\right\rangle_{i=1}^{\infty} \subset \mathcal{A}$ and $A_{i} \cap A_{j}=\emptyset$ for $i \neq j$ imply $\bigcup_{n=1}^{\infty} A_{n} \in \mathcal{A}$.

In the standard model, a probability measure is defined on a $\sigma$-algebra, while in the present paper, a probability measure is defined on a $\lambda$-system.

Definition 2. Let $S$ be a set and $\mathcal{A}$ be a $\lambda$-system on $S$. Consider a set function $p: \mathcal{A} \rightarrow$ $[0,1]$ and let $p$ satisfy the following property:

(p.1) If $\left\langle A_{i}\right\rangle_{i=1}^{\infty}$ is a sequence of disjoint sets in $\mathcal{A}$, then $p\left(\bigcup_{i=1}^{\infty} A_{i}\right)=\sum_{i=1}^{\infty} p\left(A_{i}\right)$.

Then $p$ is called a premeasure on $(S, \mathcal{A}) \cdot{ }^{15}$

If a premeasure $p$ on $(S, \mathcal{A})$ satisfies $p(S)=1$, then $(S, \mathcal{A}, p)$ is called a $\lambda$-system probability space. Throughout this paper, $(S, \mathcal{A}, p)$ denotes a $\lambda$-system probability space.

Some comments on Definitions 1 and 2 are in order. Conditions $(\lambda .1)$ and $p(S)=1$ imply that a DM knows what the whole space is. Conditions $(\lambda .2)$ and (p.1) imply that if she knows what $A$ is, she knows what the complement is. To put it differently, if she can assign probabilities to an event, she can assign probabilities to its complement.

\footnotetext{
${ }^{15}$ Formally speaking, a premeasure is defined on an algebra, not on a $\lambda$-system. For example, see Folland (1999).
} 
Conditions ( $\lambda .3)$ and (p.1) imply that if she knows what $A_{1}$ and $A_{2}$ are, she knows what the disjoint union of them is. In other words, if she can assign probabilities to disjoint sets $A_{1}, A_{2}$, respectively, she can assign probabilities to its disjoint union. On the other hand, as we see in the previous subsection, she could not assign probabilities to intersections of any sets in $\mathcal{A}$. Thus, a $\lambda$-system can be considered as a collection of subsets that she could assign probabilities to "unambiguously," in other words, can be considered as a collection of "unambiguous events." Following Epstein (1999), this paper uses a $\lambda$-system $\mathcal{A}$ for a collection of unambiguous events. Every subset of $S$ outside $\mathcal{A}$ is called ambiguous events. ${ }^{16}$ Next, we consider a set function to capture DM's attitude toward ambiguity.

Definition 3. A set function $p_{\mathcal{A}}: 2^{S} \rightarrow[0,1]$ defined by

$$
\left(\forall B \in 2^{S}\right) \quad p_{\mathcal{A}}(B) \equiv \sup \{p(A) \mid A \in \mathcal{A}, A \subset B\}
$$

is the inner measure corresponding to $(S, \mathcal{A}, p)$.

Since $\emptyset \in \mathcal{A}, p_{\mathcal{A}}$ is well-defined. Note that a DM whose beliefs are captured by $p_{\mathcal{A}}$ is very cautious. Similarly, the outer measure corresponding to $(S, \mathcal{A}, p)$ is defined as follows : A set function $p^{\mathcal{A}}: 2^{S} \rightarrow[0,1]$ defined by

$$
\left(\forall B \in 2^{S}\right) \quad p^{\mathcal{A}}(B) \equiv \inf \{p(A) \mid A \in \mathcal{A}, B \subset A\}
$$

is the outer measure corresponding to $(S, \mathcal{A}, p)$. Since $S \in \mathcal{A}, p^{\mathcal{A}}$ is also well-defined. Now, we must check what conditions the inner and outer measures satisfy.

Lemma 1. The inner and outer measures, $p_{\mathcal{A}}$ and $p^{\mathcal{A}}$ are non-additive measures.

Proof. See Appendix.

Note that $p_{\mathcal{A}}$ is not necessarily a convex non-additive measure. Since the convexity of nonadditive measures has been assumed in order to capture DM's attitude toward uncertainty, its non-convexity of non-additive measures is one of the starkest contrasts to approaches adopted in the literature on $\mathrm{CEU}$ and its applications. However, the inner measure has useful results.

\footnotetext{
${ }^{16}$ Epstein and Zhang (2001) define ambiguous events in terms of DM's preferences.
} 
Lemma 2. Let $p_{\mathcal{A}}$ and $p^{\mathcal{A}}$ be the inner and outer measures corresponding to $(S, \mathcal{A}, p)$.

(a) $p_{\mathcal{A}}$ is the conjugate of $p^{\mathcal{A}}$, that is, for all $B \in 2^{S}, p_{\mathcal{A}}(B)=1-p^{\mathcal{A}}\left(B^{c}\right)$.

(b) $p_{\mathcal{A}}$ is superadditive, that is, for all $B_{1}, B_{2} \in 2^{S}$ such that $B_{1} \cap B_{2}=\emptyset, p_{\mathcal{A}}\left(B_{1} \cup B_{2}\right) \geq$ $p_{\mathcal{A}}\left(B_{1}\right)+p_{\mathcal{A}}\left(B_{2}\right)$.

Proof. See Appendix.

Recall that the inner and outer measures are non-additive measures. The non-additivity provides the next lemma.

Lemma 3. Let $p_{\mathcal{A}}$ and $p^{\mathcal{A}}$ be the inner and outer measures corresponding to $(S, \mathcal{A}, p)$. Then for all $X \in B(S, \mathbb{R})$,

$$
\int X(s) p_{\mathcal{A}}(d s) \leq \int X(s) p^{\mathcal{A}}(d s)
$$

Proof. See Appendix.

\section{Portfolio Selection Problems}

In this section, we derive our main results. Under CEU with the inner measure $p_{\mathcal{A}}$, we show that there exists a range of prices, in which investors neither buy nor sell.

First, we consider the portfolio selection problem à la Arrow (1965) and provide one of the main results of this paper. Let $W \in \mathbb{R}_{+}$be the wealth at $t=0, N \in \mathbb{R}$ be the amount of money invested at $t=0, q>0$ be the price of a risky asset at $t=0$, and $X$ be the random payoff of the asset at $t=1$, where $X \in B(S, \mathbb{R})$. Moreover, let $u: \mathbb{R} \rightarrow \mathbb{R}$ be a monotonic increasing concave function. ${ }^{17}$

An investor is assumed to invest in two assets, a riskless asset and a risky asset. The problem is to choose $N$ so as to maximize

$$
\int u(W-N+(N / q) X(s)) p_{\mathcal{A}}(d s)
$$

In other words, she chooses the amount of money $N$ to invest the risky asset in order to maximize her non-expected utility of the terminal wealth.

\footnotetext{
${ }^{17}$ In this paper, we capture investor's attitude toward risk by $u$.
} 
Theorem 1. Let $p_{\mathcal{A}}$ and $p^{\mathcal{A}}$ be the inner and outer measures corresponding to $(S, \mathcal{A}, p)$. Then, this investor will neither buy nor sell the risky asset, if q satisfies:

$$
\int X(s) p_{\mathcal{A}}(d s)<q<\int X(s) p^{\mathcal{A}}(d s)
$$

Proof. See Appendix.

This theorem states that the range she will neither buy nor sell the risky asset does not depend on her attitude toward risk measured by $u$. Moreover, the range does not depend on her attitude toward uncertainty captured by the convexity of non-additive measures. In our setting, the range is determined by a set of unambiguous events $\mathcal{A}$, and the inner and outer measures $p_{\mathcal{A}}$ and $p^{\mathcal{A}}$ corresponding to $(S, \mathcal{A}, p)$.

Before we investigate a possibility of the further application of the ambiguity approach, we provide an example.

Example 3.1. Suppose that two investors consider trading the stock of GE whose current price is $\$ 35$ per share. On the one hand, one investor might want to sell it if the stock price is over $\$ 40$, and might want to buy it if the stock price is below $\$ 30$. On the other hand, the other investor might want to sell it if the stock price is over $\$ 38$, and might want to buy it if the stock price is below $\$ 32$. In this case, they will never consider changing their positions. Moreover, ceteris paribus, suppose that the stock's current price is $\$ 39$. In this case, the first investor does not consider trading the stock. However, the second one does sell it.

While such a situation is not actually observable, it would cause no trouble to suppose that different investors behave in a different way. To put it differently, it is natural to suppose that a spread between the striking price for selling and that for buying is not unique among different investors. What brings the differences of the spreads between the striking price for selling and that for buying among investors? This phenomenon as well as the existence of portfolio inertia cannot be accounted for under the standard expected utility theory. However, adopting the ambiguity approach enables us to obtain a solution to the problem. Before we present the last theorem of this paper, some mathematical 
results are in order. Let $\left(S, \mathcal{A}_{1}, p_{1}\right)$ and $\left(S, \mathcal{A}_{2}, p_{2}\right)$ be $\lambda$-system probability spaces, where $\mathcal{A}_{1} \subset \mathcal{A}_{2}$, and $p_{1}=\left.p_{2}\right|_{\mathcal{A}_{1}}$, i.e., $p_{1}$ is the restriction of $p_{2}$ to $\mathcal{A}_{1}$. Now, we are in a position to present two results, which play a crucial part in obtaining the last result of this paper.

Lemma 4. Let $\left(S, \mathcal{A}_{1}, p_{1}\right)$ and $\left(S, \mathcal{A}_{2}, p_{2}\right)$ be $\lambda$-system probability spaces, where $\mathcal{A}_{1} \subset \mathcal{A}_{2}$, and $p_{1}=\left.p_{2}\right|_{\mathcal{A}_{1}}$. Then, for all $B \in 2^{S}, p_{\mathcal{A}_{1}}(B) \leq p_{\mathcal{A}_{2}}(B)$ and $p^{\mathcal{A}_{2}}(B) \leq p^{\mathcal{A}_{1}}(B)$.

For the inner measure, Lemma 4 states that a fine $\lambda$-system yields a fine assessment to all the subsets outside $\mathcal{A}$ by approximating them from the inside. For the outer measure, Lemma 4 states that a fine $\lambda$-system yields a fine assessment to all the subsets outside $\mathcal{A}$ by approximating them from the outside. From above observations, $p_{\mathcal{A}}$ can be considered as a set function to capture a form of aversion to ambiguity through $\mathcal{A}$.

Theorem 2. Let $\left(S, \mathcal{A}_{1}, p_{1}\right)$ and $\left(S, \mathcal{A}_{2}, p_{2}\right)$ be $\lambda$-system probability spaces, where $\mathcal{A}_{1} \subset$ $\mathcal{A}_{2}, p_{1}=\left.p_{2}\right|_{\mathcal{A}_{1}}$, and $X \in B(S, \mathbb{R})$. Then

$$
\int X(s) p_{\mathcal{A}_{1}}(d s) \leq \int X(s) p_{\mathcal{A}_{2}}(d s) \text { and } \int X(s) p^{\mathcal{A}_{2}}(d s) \leq \int X(s) p^{\mathcal{A}_{1}}(d s) .
$$

Proof. See Appendix.

This theorem states that the Choquet integral with respect to $p_{\mathcal{A}}$ is increasing in $\mathcal{A}$, and that the Choquet integral with respect to $p^{\mathcal{A}}$ is decreasing in $\mathcal{A}$. In other words, the monotonicity of the Choquet integral with respect to $\mathcal{A}$ provides a fine evaluation of $X(s)$.

Theorems 1 and 2 provide the last theorem of this paper.

Theorem 3. Let $\left(S, \mathcal{A}_{1}, p_{1}\right)$ and $\left(S, \mathcal{A}_{2}, p_{2}\right)$ be $\lambda$-system probability spaces, where $\mathcal{A}_{1} \subset$ $\mathcal{A}_{2}$, and $p_{1}=\left.p_{2}\right|_{\mathcal{A}_{1}}$, and let $q$ be the price of a risky asset at $t=0$, and let $X \in B(S, \mathbb{R})$ be the asset price at $t=1$. Suppose that one investor's preferences are represented by $C E U$ with the inner measure $p_{\mathcal{A}_{1}}$ corresponding to $\left(S, \mathcal{A}_{1}, p_{1}\right)$, and the other investor's preferences are represented by $C E U$ with the inner measure $p_{\mathcal{A}_{2}}$ corresponding to $\left(S, \mathcal{A}_{2}, p_{2}\right)$. Then both investors neither buy nor sell the asset if $q$ satisfies:

$$
\int X(s) p_{\mathcal{A}_{2}}(d s)<q<\int X(s) p^{\mathcal{A}_{2}}(d s)
$$


Furthemore, for such an asset price, the spread between the striking price for selling and that for buying with respect to $\mathcal{A}_{1}$ is wider than that with respect to $\mathcal{A}_{2}$, i.e.,

$$
\int X(s) p_{\mathcal{A}_{1}}(d s) \leq \int X(s) p_{\mathcal{A}_{2}}(d s)<q<\int X(s) p^{\mathcal{A}_{2}}(d s) \leq \int X(s) p^{\mathcal{A}_{1}}(d s) .
$$

The latter part of this theorm provides a solution to the problem: what leads to the differences of the spreads between the striking price for selling and that for buying among different investors? If an investor has more events to which she assigns probabilities, then the spread shrinks compared with the case in which she has less events to which she assigns probabilities. This interpretation is made possible by capturing ambiguity through $\lambda$-systems. ${ }^{18}$

\section{Conclusion}

This paper adopts the ambiguity approach to show that there exists portfolio inertia under CEU with an inner measure. A related work is Dow and Werlang (1992) who adopt the uncertainty approach to obtain a result similar to ours. However, there are two differences between their paper and this one. First, their model is based on CEU with every convex non-additive measure. On the other hand, we adopt CEU with the inner measure $p_{\mathcal{A}}$, which is neither additive nor convex. As the example in Subection 2.2 shows, there exists some situation where the convexity of non-additive measures does not account for investor's behavior. This implies that such a situation cannot be accounted for within the framework of theirs, and that the convexity assumption is not crucial for the existence of portfolio inertia. Second, we introduce the concept of ambiguity to portfolio selection problems by letting $\lambda$-systems serve as domains of probabilities. Adopting the concept of ambiguity enables us to account for a problem that cannot be explained by Dow and Werlang (1992). To the best of my knowledge, analyses based on the ambiguity approach have not been done in the fields of game theory or finance. Our results provide a new view

\footnotetext{
${ }^{18} \mathrm{~A}$ different approach appears in Dow and Werlang (1992). Let $\mu, \mu_{1}$ and $\mu_{2}$ be non-additive measures on $\left(S, 2^{S}\right)$. They define uncertainty aversion, by $c(\mu, A)=1-\mu(A)-\mu\left(A^{c}\right)$, measured by $\mu$, for every set $A$ in $2^{S}$. Furthermore, they say that $\mu_{2}$ is more uncertainty averse than $\mu_{1}$ if $c\left(\mu_{2}, A\right) \geq c\left(\mu_{1}, A\right)$ for every set $A$ in $2^{S}$. Based on this definition, they show that if $\mu_{2}$ is more uncertainty averse than $\mu_{1}$, a spread between the striking price for selling and that for buying expands.
} 
point by illuminating the difference between the uncertainty approach and the ambiguity approach. Furthemore, our results imply that the convexity of non-additive measures is not a panacea for analyzing DM's behavior under uncertainty. If this paper becomes a first step toward the further research on the ambiguity approach, the goal of this paper will be achieved. 


\section{Appendix}

\section{A. Mathematical Results}

In this subsection, let $\mu$ be a non-additive measure on $\left(S, 2^{S}\right)$.

\section{Fact 1.}

$$
(\forall X, Y \in B(S, \mathbb{R})) X \geq Y \Rightarrow \int X(s) \mu(d s) \geq \int Y(s) \mu(d s)
$$

\section{Fact 2.}

$$
(\forall X \in B(S, \mathbb{R}))(\forall a \in \mathbb{R})\left(\forall b \in \mathbb{R}_{+}\right) \int(a+b X(s)) \mu(d s)=a+b \int X(s) \mu(d s) .
$$

\section{Fact 3.}

$$
(\forall X \in B(S, \mathbb{R})) \int X(s) \mu(d s)=-\int-X(s) \mu^{\prime}(d s),
$$

where $\mu^{\prime}$ is the conjugate of $\mu$.

Lemma 5. Let $u: \mathbb{R} \rightarrow \mathbb{R}$. If $u$ is concave on $(a, b)$ and $t_{0} \in(a, b)$, then there exists $\beta \in \mathbb{R}$ such that

$$
u(t) \leq u\left(t_{0}\right)+\beta\left(t-t_{0}\right) \text { for all } t \in(a, b) .
$$

For such a $\beta$, the next inequlities hold:

$$
\lim _{t \downarrow t_{0}} \frac{u(t)-u\left(t_{0}\right)}{t-t_{0}} \equiv u_{+}^{\prime}\left(t_{0}\right) \leq \beta \leq \lim _{t \uparrow t_{0}} \frac{u(t)-u\left(t_{0}\right)}{t-t_{0}} \equiv u_{-}^{\prime}\left(t_{0}\right) .
$$

Furtheremore, if $u$ is increasing on $(a, b)$, then $0 \leq u_{+}^{\prime}\left(t_{0}\right)$.

Proof of Lemma 5. For the first claim, see Folland (1999, p.109), or Royden (1987, p.115). The second and third ones are immediate. 


\section{B. Proofs}

Proof of Lemma 1. For all $B_{1}, B_{2} \in 2^{S}$, let $B_{1} \subset B_{2}$. Then $\left\{p(A) \mid A \in \mathcal{A}, A \subset B_{1}\right\} \subset$ $\left\{p(A) \mid A \in \mathcal{A}, A \subset B_{2}\right\}$, which implies $p_{\mathcal{A}}\left(B_{1}\right) \leq p_{\mathcal{A}}\left(B_{2}\right)$. The proof that $p_{\mathcal{A}}(\emptyset)=0$ is trivial.

Proof of Lemma 2. (a)

$$
\begin{aligned}
\left(\forall B \in 2^{S}\right) \quad p_{\mathcal{A}}(B) & =\sup \{p(A) \mid A \in \mathcal{A}, A \subset B\} \\
& =-\inf \{-p(A) \mid A \in \mathcal{A}, A \subset B\} \\
& =-\inf \left\{p\left(A^{c}\right)-1 \mid A^{c} \in \mathcal{A}, B^{c} \subset A^{c}\right\} \\
& =1-\inf \left\{p\left(A^{c}\right) \mid A^{c} \in \mathcal{A}, B^{c} \subset A^{c}\right\} \\
& =1-p^{\mathcal{A}}\left(B^{c}\right) .
\end{aligned}
$$

(b) If $\varepsilon>0$, there exist $A_{1}, A_{2} \in \mathcal{A}, A_{1} \subset B_{1}, A_{2} \subset B_{2}, B_{1} \cap B_{2}=\emptyset$ such that $p_{\mathcal{A}}\left(B_{1}\right)-$ $\varepsilon \leq p\left(A_{1}\right)$ and $p_{\mathcal{A}}\left(B_{2}\right)-\varepsilon \leq p\left(A_{2}\right)$. So

$$
\begin{aligned}
p_{\mathcal{A}}\left(B_{1}\right)+p_{\mathcal{A}}\left(B_{2}\right)-2 \varepsilon & \leq p\left(A_{1}\right)+p\left(A_{2}\right) \\
& =p\left(A_{1} \cup A_{2}\right) \\
& \leq p_{\mathcal{A}}\left(B_{1} \cup B_{2}\right) .
\end{aligned}
$$

The first equality follows from the facts that $B_{1} \cap B_{2}=\emptyset \Rightarrow A_{1} \cap A_{2}=\emptyset, A_{1} \cup A_{2} \in \mathcal{A}$ and $p$ is a premeasure on $\mathcal{A}$. The last inequality holds by the definition of $p_{\mathcal{A}}$. Since $\varepsilon$ is arbitrary, the proof is completed.

Proof of Lemma 3. This follows from Fact 1 and the fact that $p_{\mathcal{A}}(B) \leq p^{\mathcal{A}}(B)$ for all $B \in 2^{S}$. 
Theorem 4 (Jensen's Inequality). Let $\left(S, 2^{S}, \mu\right)$ be a measure space with $\mu(S)=1$, where $\mu$ is a non-additive measure. Let $X: S \rightarrow(a, b)$ be in $B(S, \mathbb{R})$, and $u$ be a concave, increasing and real-valued function on $(a, b)$. Then for all non-additive measures $\mu$,

$$
\int u(X(s)) \mu(d s) \leq u\left(\int X(s) \mu(d s)\right) .
$$

Proof. Let $t_{0}=\int X(s) \mu(d s)$ and $t=u(s)$. Then $t_{0} \in(a, b)$ and $t \in(a, b)$. By Lemma 5 , there exists $\beta \in\left[u_{+}^{\prime}\left(t_{0}\right), u_{-}^{\prime}\left(t_{0}\right)\right] \subset \mathbb{R}_{+}$such that

$$
u(X(s)) \leq u\left(\int X(s) \mu(d s)\right)+\beta\left(X(s)-\int X(s) \mu(d s)\right) \text { for all } X(s) \in(a, b)
$$

Integrating both sides with respect to $\mu$ and Fact 1 imply

$$
\begin{aligned}
& \int u(X(s)) \mu(d s) \\
\leq & \int\left(u\left(\int X(s) \mu(d s)\right)+\beta\left(X(s)-\int X(s) \mu(d s)\right)\right) \mu(d s) .
\end{aligned}
$$

Then

$$
\begin{aligned}
& \int u(X(s)) \mu(d s) \\
\leq & u\left(\int X(s) \mu(d s)\right)+\beta \int\left(X(s)-\int X(s) \mu(d s)\right) \mu(d s) \\
= & u\left(\int X(s) \mu(d s)\right)+\beta\left(\int X(s) \mu(d s)-\int X(s) \mu(d s)\right) \\
= & u\left(\int X(s) \mu(d s)\right) .
\end{aligned}
$$

The inequality and the first equality follow from Fact 2.

Proof of Theorem 1. Assume

$$
\int X(s) p_{\mathcal{A}}(d s)<q
$$

Then

$$
\begin{aligned}
(\forall N \geq 0) & \int u(W-N+(N / q) X(s)) p_{\mathcal{A}}(d s) \\
\leq & u\left(\int(W-N+(N / q) X(s)) p_{\mathcal{A}}(d s)\right)
\end{aligned}
$$




$$
\begin{aligned}
& =u\left(W-N+(N / q) \int X(s) p_{\mathcal{A}}(d s)\right) \\
& <u(W-N+(N / q) q) \\
& =u(W) .
\end{aligned}
$$

The first inequality follows from Theorem 4. The first equality holds by Fact 2 . The second inequality follows from the assumption.

Next, assume

$$
q<\int X(s) p^{\mathcal{A}}(d s)
$$

Similarly,

$$
\begin{aligned}
(\forall N \leq 0) & \int u(W-N+(N / q) X(s)) p_{\mathcal{A}}(d s) \\
\leq & u\left(\int(W-N+(N / q) X(s)) p_{\mathcal{A}}(d s)\right) \\
= & u\left(W-N-(N / q) \int(-X(s)) p_{\mathcal{A}}(d s)\right) \\
= & u\left(W-N-(N / q)\left(-\int X(s) p^{\mathcal{A}}(d s)\right)\right) \\
< & u(W-N-(N / q)(-q)) \\
= & u(W) .
\end{aligned}
$$

The first inequality holds by Theorem 4. The first equality holds by Fact 2. The second equality holds by Fact 3. The last inequality follows from the assumption.

Proof of Theorem 2. The first and second inequalities follow from the boundedness of $X$, the definition of the Choquet integral, and Lemmas 3 and 4. 


\section{References}

Anscombe, F. and R. J. Aumann (1963): "A Definition of Subjective Probability," Annals of Mathematical Statistics 34, 199-205.

Arrow, K. J. (1965): Aspects of the Theory of Risk-Bearing. Helsinki: Yrjo Jahnsonin Saatio.

Casadesus-Masanell, R., P. Klibanoff and E. Ozdenoren (2000): "Maxmin Expected Utility over Savage Acts with a Set of Priors," Journal of Economic Theory 92, $35-65$.

Dow, J. and S. R. C. Werlang (1992): "Uncertainty Aversion, Risk Aversion, and the Optimal Choice of Portfolio," Econometrica 60, 197-204.

Dow, J. and S. R. C. Werlang (1994): "Nash Equilibrium under Knightian Uncertainty: Breaking Down Backward Induction," Journal of Economic Theory 64, 305-324.

Ellsberg, D. (1961): "Risk, Ambiguity, and the Savage Axioms," Quarterly Journal of Economics 75, 643-669.

Epstein, L. G. (1999): “A Definition of Uncertainty Aversion," Review of Economic Studies 66, 579-608.

Epstein, L. G. and T. Wang (1994): "Intertemporal Asset Pricing under Knightian Uncertainty," Econometrica 62, 283-322.

Epstein, L. G. and T. Wang (1995): "Uncertainty, Risk-Neutral Measures and Security Price Booms and Crashes," Journal of Economic Theory 67, 40-82.

Epstein, L. G. and J. Zhang (1999): "Least Convex Capacities," Economic Theory $13,263-286$.

Epstein, L. G. and J. Zhang (2001): "Subjective Probabilities on Subjective Unambiguous Events," Econometrica 69, 265-306.

Folland, G. B. (1999): Real Analysis, Second Edition. New York: Wiley.

Ghirardato, P. (1994): "Agency Theory with Uncertainty Aversion," mimeo. 
Ghirardato, P. (1997): "On Independence for Non-Additive Measures, with a Fubini Theorem," Journal of Economic Theory 73, 261-291.

Ghirardato, P., P. Klibanoff and M. Marinacci (1998): "Additivity with Multiple Priors," Journal of Mathematical Economics 30, 405-420.

Ghirardato, P. and M. Marinacci (2002): "Ambiguity Made Precise: A Comparative Foundation," Journal of Economic Theory 102, 251-289.

Gilboa, I. (1987): "Expected Utility with Purely Subjective Non-Additive Probabilities," Journal of Mathematical Economics 16, 65-88.

Gilboa, I. and D. Schmeidler (1989): "Maxmin Expected Utility with Non-Unique Priors," Journal of Mathematical Economics 18, 141-153.

Knight, F. (1921): Risk, Uncertainty and Profit. Boston: Houghton Mifflin.

Lo, K. C. (1996): "Equilibrium in Beliefs under Uncertainty," Journal of Economic Theory 71, 443-484.

Lo, K. C. (1998): "Sealed Bid Auctions with Uncertainty Averse Bidders," Economic Theory 12, 1-20.

Lo, K. C. (1999): "Extensive Form Games with Uncertainty Averse Players," Games and Economic Behavior 28, 256-270.

Marinacci, M. (2000): "Ambiguous Games," Games and Economic Behavior 31, 191-219.

Royden, H. L. (1987): Real Analysis, Third Edition. Prentice Hall.

Savage, L. J. (1972): The Foundation of Statistics. Dover Publications. (First published 1954.)

Schmeidler, D. (1989): "Subjective Probability and Expected Utility without Additivity," Econometrica 57, 571-587.

Zhang, J. (1999): "Qualitative Probabilities on $\lambda$-systems," Mathematical Social Sciences 38, 11-20. 
Zhang, J. (2002): "Subjective Ambiguity, Expected Utility and Choquet Expected Utility," Economic Theory 20, 159-181. 